\title{
Protection du littoral contre l'érosion marine : application au tombolo Ouest de Giens
}

\author{
Pierre FARNOLE ${ }^{1}$, Jean BOUGIS ${ }^{2}$, M. RITONDALE ${ }^{3}$, M. BARBARROUX ${ }^{4}$ \\ ${ }^{1}$ Directeur ERAMM - (Im. Thélème 1503, rte des Dolines - BP42 06901 Sophia \\ Antipolis; tél.:0492969205 (Eramm.Farnole@wanadoo.fr) \\ ${ }^{2}$ Ingénieur Conseil 34, ch. du Moulin 06650 Opio; Tél : 0493777422 \\ (ibougis@aws.fr) \\ ${ }^{3}$ Maire de la commune d'Hyères (Hôtel de Ville - BP709 - 83412 Hyères) \\ ${ }^{4}$ Adjoint à l'Environnement (Hôtel de Ville - BP709 - 83412 Hyères)
}

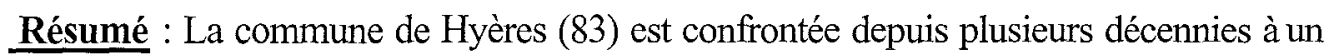
phénomène d'érosion sur le cordon littoral Ouest du tombolo de Giens. Dans les années 1970, BLANC avait montré que le secteur le plus fragile était la partie centrale, mais depuis quelques années, le problème d'érosion s'est décalé dans la partie Nord du tombolo. Bien que de nombreuses études aient été engagées, aucune n'a permis d'aboutir à des solutions concrètes car l'état des connaissances sur les processus hydrosédimentaires était insuffisant. L'objectif de cette communication est de présenter comment à partir des données disponibles, de l'observation et de la modélisation de l'agitation, il a été possible d'identifier les causes de ce déséquilibre et d'y proposer des remèdes. Une des causes originelles est attribuée à des extractions abusives de granulats au début du siècle qui auraient provoqué un déséquilibre morphosédimentaire des petits fonds, comme le démontre la comparaison des levés bathymétriques anciens et actuels.

Abstract : Hyères city is submitted to coastal erosion on the shore zone of Almanarre beach. In 1970, BLANC have showed the shore fragility of the central west tombolo. Most of some years erosion processes have migrated on the northern part of the tombolo. Several studies don't permit to solve the problem because the knowledge of sedimentary processes was insufficient. The aim of this paper is to demonstrate with pluri-disciplinaries studies, the contribution of in-situ measurement (wave, current, sediments..) and wave models in studying causes of the shore erosion.

\section{Introduction}

La commune d'Hyères a engagé depuis une trentaine d'années de nombreuses études pour tenter d'endiguer le phénomène d'érosion que subit la plage de l'Almanarre et plus généralement l'écosystème côtier du Golfe de Giens. A ce jour, aucune des études n'a permis d'aboutir à des solutions concrètes d'aménagement pour stabiliser le recul du trait de côte. Il s'est avéré que l'état des connaissances a été jugé insuffisant, en particulier, sur les aspects hydrosédimentaires. Pour y remédier, la commune a souhaité engager une nouvelle étude localisée sur la plage de l'Almanarre, située au Nord-Est du Golfe de Giens (Figure 1). Celle-ci s'appuie sur une double approche de mesures in-situ 
(bathymétrie et sédimentologie; levé géotechnique, houle et courants), et de modélisations de l'agitation (modèle d'approche et modèle rapproché), pour préciser localement l'incidence de la houle et des courants induits sur l'équilibre de la plage. Dans ce cadre, la commune de Hyères-les-Palmiers a confié au cabinet ERAMM associé au cabinet Jean BOUGIS, le soin de déterminer les causes du phénomène d'érosion et d'y apporter des solutions adaptées.

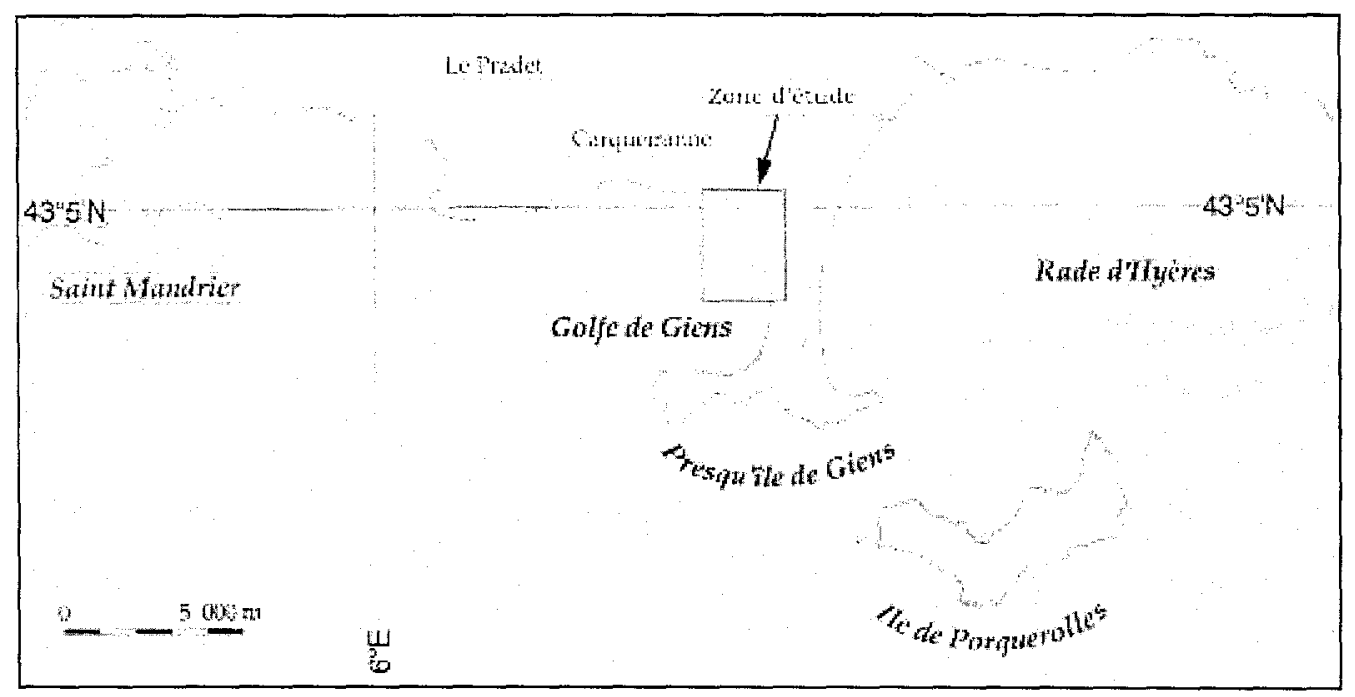

Figure 1: plan de situation - localisation de la plage de 1'Almanarre ; Figure 1: Located area

\section{Historique}

\subsection{La dynamique littorale}

Le site du tombolo de Giens a fait l'objet de très nombreuses études, particulièrement sur les aspects écologiques concernant les phanérogames marines et la végétation dunaire qui ont été traités de façon plus précise et plus approfondie que les aspects physiques relatifs à la dynamique littorale et à la morphologie des petits fonds. Les photos aériennes $\mathrm{du}$ début du siècle montrent que la plage de l'Almanarre était stable voire en engraissement. Plus récemment, BLANC [1] étudie en détail la dynamique Ouest de Giens (Figure 2) et montre des processus d'érosion dans la partie centrale et Nord du tombolo Ouest. Il souligne l'existence de chenaux perpendiculaires à la ligne de rivage, creusés par des courants de décharge qui transporteraient les sédiments du prisme littoral vers les fonds de $-5 \mathrm{~m}$ à $-10 \mathrm{~m}$, en dehors de la zone d'échange sédimentaire du tombolo, contribuant ainsi à la perte des sédiments du cordon littoral. 


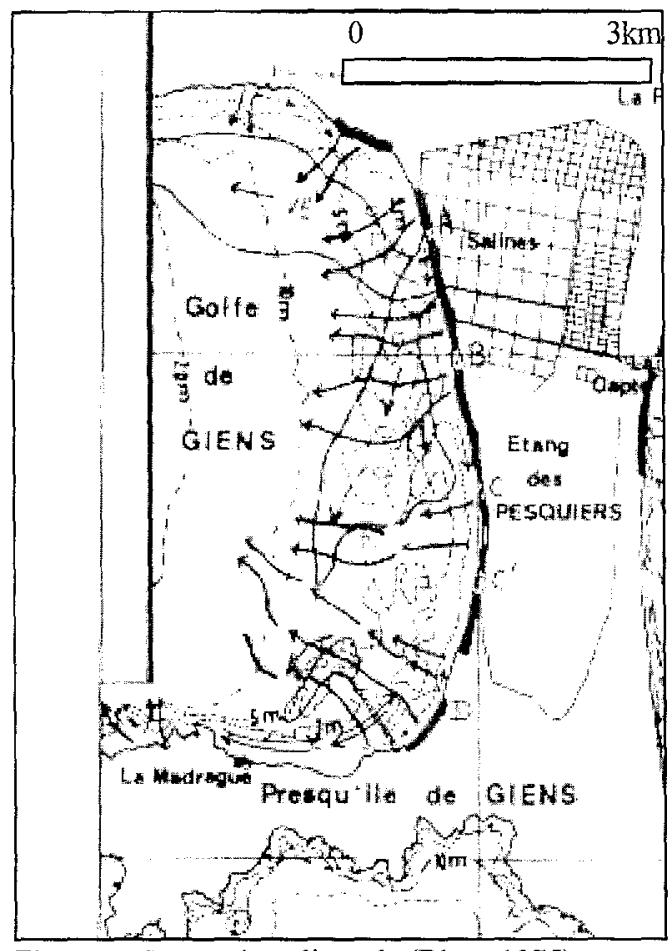

Figure 2 : Dynamique littorale (Blanc 1975)

\subsection{Evolutions bathymétriques}

Le levé de 1896 du SHOM peut être considéré comme une situation référence car la plage de l'Almanarre en pente douce à $1 \%$ sur un linéaire de $400 \mathrm{~m}$ était en équilibre entre les bornes 7 et 10 (Figure 3). Cette situation est très différente du levé actuel où, l'on relève un creusement des fonds à seulement $100 \mathrm{~m}$ du trait de côte avec des profondeurs atteignant $-5 \mathrm{~m}$ à $-6 \mathrm{~m}$ (Figure 4). La comparaison des levés anciens et montre que la brèche et les trous observés ne sont pas des formes reliques, mais qu'ils se sont creusés avec le temps. Il existe une forte probabilité pour que les extractions anciennes soient à l'origine de ce creusement sur plusieurs mètres

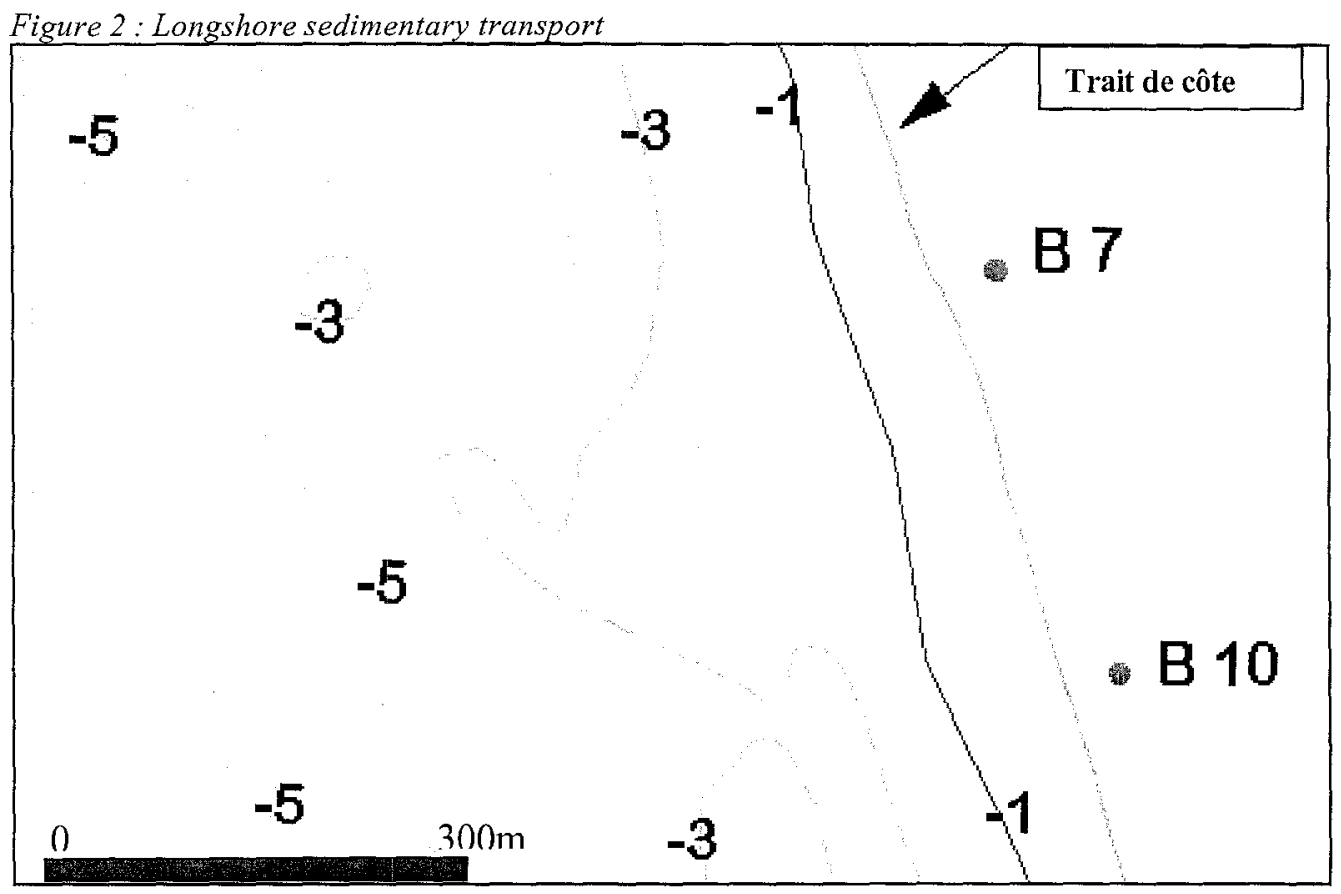

Figure 3 : bathymétrie de 1896 - (source SHOM) - Figure 3 : bathymetry in 1896

d'épaisseur. En 1896, la largeur d'estran (50m environ) rendait aisée toute pratique d'extraction depuis la plage elle-même dont la granulométrie des sables et graviers constituait un matériel de choix pour la fabrication du béton. 


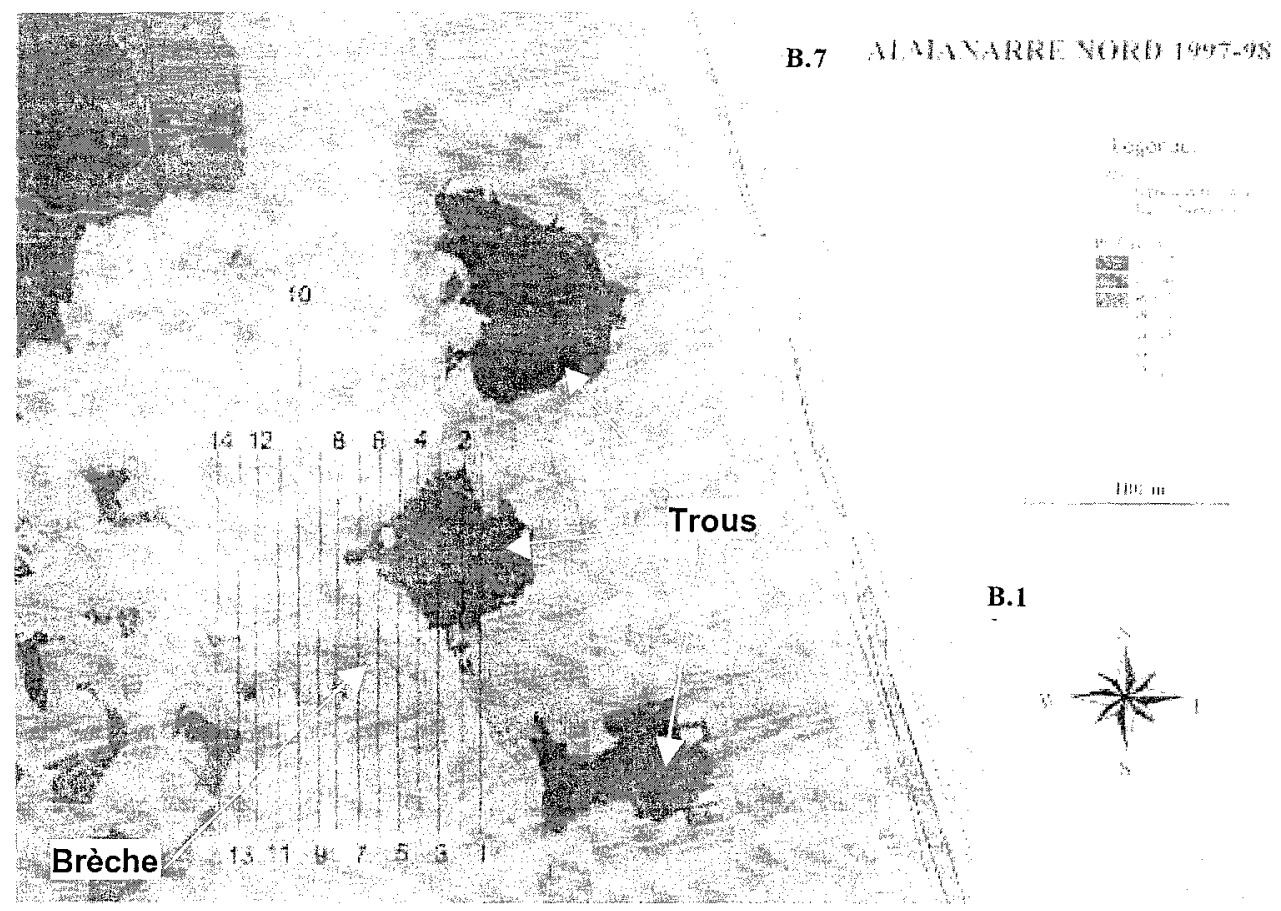

Figure 4 : Bathymétrie de la plage de l'Almanarre entre les bornes 7 et 10 (levé Eol de 1997-98)

Figure 4: Almanarre Beach bathymetry within hectometric stone 7 and 10

En 1998, le niveau s'est creusé de plus de $2 \mathrm{~m}$ à $100 \mathrm{~m}$ du trait de côte et bien plus audelà Le stade d'évolution actuel est préoccupant car le platier gréseux qui assure la dernière protection de la plage est très érodé. Avec le temps, le creusement de la brèche et des trous se poursuit en profondeur et latéralement sans que l'on puisse présager d'un ralentissement ou d'une stabilisation de cette évolution.

\subsection{Sédimentologie et géotechnique}

Les investigations sédimentologiques et géotechniques menées par la société ERG [3] ont permis de préciser la nature, la structure et la résistance du substratum sous-marin au niveau des dépressions (brèche et trous) :

1. Le banc gréseux, situé à $200 \mathrm{~m}$ du trait de côte suivant un alignement NordSud, est composé d'une dalle large de $50 \mathrm{~m}$, limitée par un talus haut de $2 \mathrm{~m}$. $\mathrm{Au}$ niveau de la brèche, le substratum est composé par un grès roux conglomératique au faciès altéré et poreux. La roche subit une biodégradation qui s'ajoute aux érosions liées aux agents hydrodynamiques. Dans les dépressions, de part et d'autre du platier, on note la présence de sables, de graviers et galets quartzeux provenant du démantèlement du platier.

2. Les sables sont très mobiles comme en témoigne la présence de ripple-marks parallèles à la côte qui traduisent un transport onshore-offshore. 
3. Les argiles noires peu consolidées situées près de la côte contiennent de nombreux débris coquilliers dans leur couche supérieure. La couleur de ces argiles ainsi que la position de certaines coques fossiles (les deux valves réunies) indiquent un dépôt Quaternaire dans un milieu réducteur et calme.

4. Les argiles molles calcaréo-sableuses tapissent les dépressions dans une zone large de $100 \mathrm{~m}$ entre les argiles noires et les grès, sous une faible épaisseur de sable.

Les essais géotechniques sur le platier gréseux indiquent une forte hétérogénéité mécanique du banc de grès due à l'altération et à la porosité de la matrice. Les essais de pénétration dynamique font ressortir un premier niveau de grés hétérogène et faiblement compact d'une épaisseur moyenne de 1,2 m, puis un second horizon de même épaisseur, induré provoquant le refus.

\subsection{Mesures in-situ de la houle et des courants de houle}

Les mesures de houle et de courant ont été réalisées à l'aide de l'Ophiure III qui est un houlographe-courantographe développé par la société Océanoservice. Cet appareil permet de mesurer les principales caractéristiques de la houle et les courants induits : hauteur, période, sens de propagation, variations du niveau d'eau, courant moyen, courant orbital, courants secondaires. Dans le cas présent, la recherche de phénomène de type rip-current nous a conduit à sélectionner ce type d'appareil qui présente plusieurs avantages car il mesure les caractéristiques de la houle $1 \mathrm{~m}$ au-dessus du fond juste avant le déferlement : houle directionnelle principale et secondaire. Lors de la campagne de mesure, un coup de mer de secteur Sud-Sud-Ouest (6/11/00) s'est manifesté avec un vent modéré de Sud $(11 \mathrm{~m} / \mathrm{s})$ et une chute de la pression barométrique à 989,5 millibars. Dans le Golfe de Giens, la houle dominante est de secteur Sud-Sud-Ouest à Sud-Ouest. La hauteur significative de la houle $\left(\mathrm{H}_{1 / 3}\right)$ maximum mesurée est de $1,40 \mathrm{~m}$ pour une période correspondante, Tpic de 9,5s.

\subsubsection{Comparaison des houles mesurées au large et à la côte en novembre 2000}

La houle au large de Porquerolles est mesurée par une bouée Datawell des Phares et Balises (Var) et traitée par le CETMEF [2]. La fréquence des enregistrements est d'une demi heure alors que L'Ophiure III mesure la houle toutes les 2 heures. Les $\mathrm{H}_{/ 3}$ maximales mesurées atteignent 4,5m au large et $1,35 \mathrm{~m}$ à la côte la journée du 6/11/01 entre 10:00 et 12:00 $\zeta$. Une évolution similaire s'observe sur toute la période de mesure du $1 / 11$ au 29/11. Un coefficient d'atténuation de la houle entre la côte et large a été calculé.

$$
C=\frac{H_{1 / 3 / \text { arge }}=}{H_{l / 3 \text { côte }}}=\frac{4,5}{1,35}=0,28
$$


En comparaison avec le modèle d'approche (Vag), on note un écart de 0,1 en moins, $C$ $=0,18$. Cet écart est sans doute lié à des effets de diffraction non pris en compte dans les calculs d'approche.

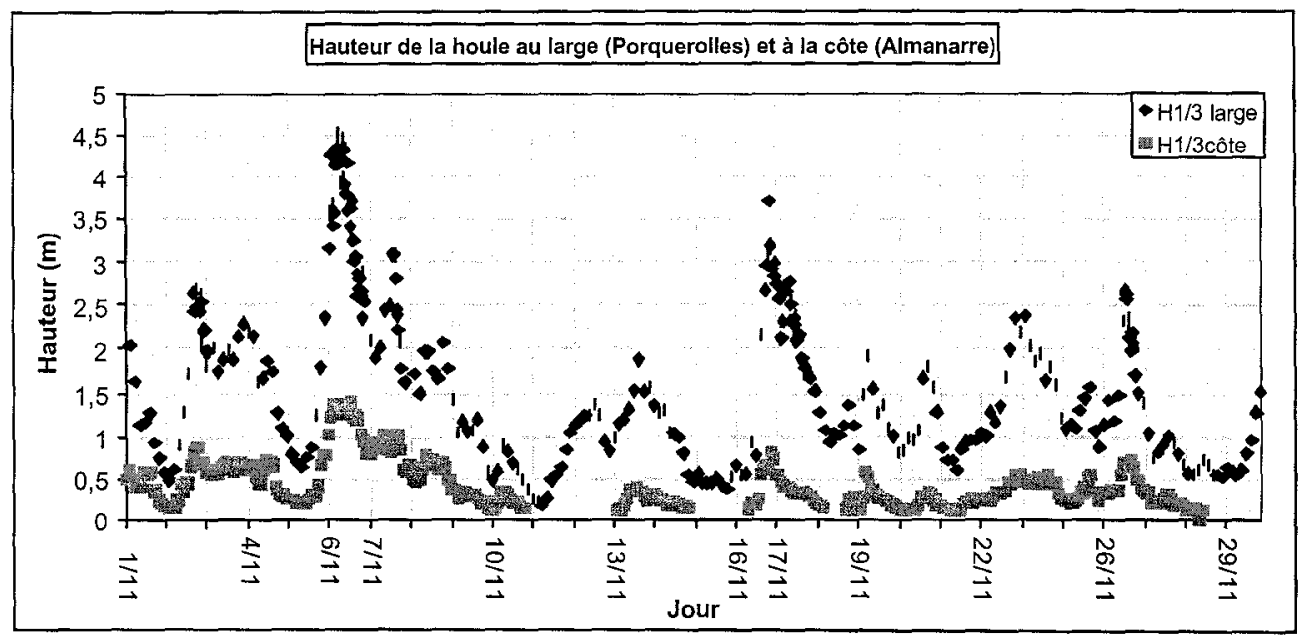

Figure 5: comparaison des hauteurs de houle au large (Porquerolles) et à la côte (Almanarre) Figure 5 : height wave comparison between the sea and the coast

\subsubsection{Direction et vitesse des courants orbitaux secondaires}

L'Ophiure III peut mesurer des courants orbitaux dont la direction est différente de celle de la houle incidente. Sur une séquence de 17', le programme sélectionne 7 vagues les plus fortes issues, d'une direction différente du train de houle. On note 3 directions de courants préférentielles apparaissent (Figure 6):

$\mathrm{N} 130^{\circ}-\mathrm{N} 190^{\circ} ; \mathrm{N} 240^{\circ}-\mathrm{N} 290^{\circ} ; \mathrm{N} 30^{\circ}-\mathrm{N} 60^{\circ}$

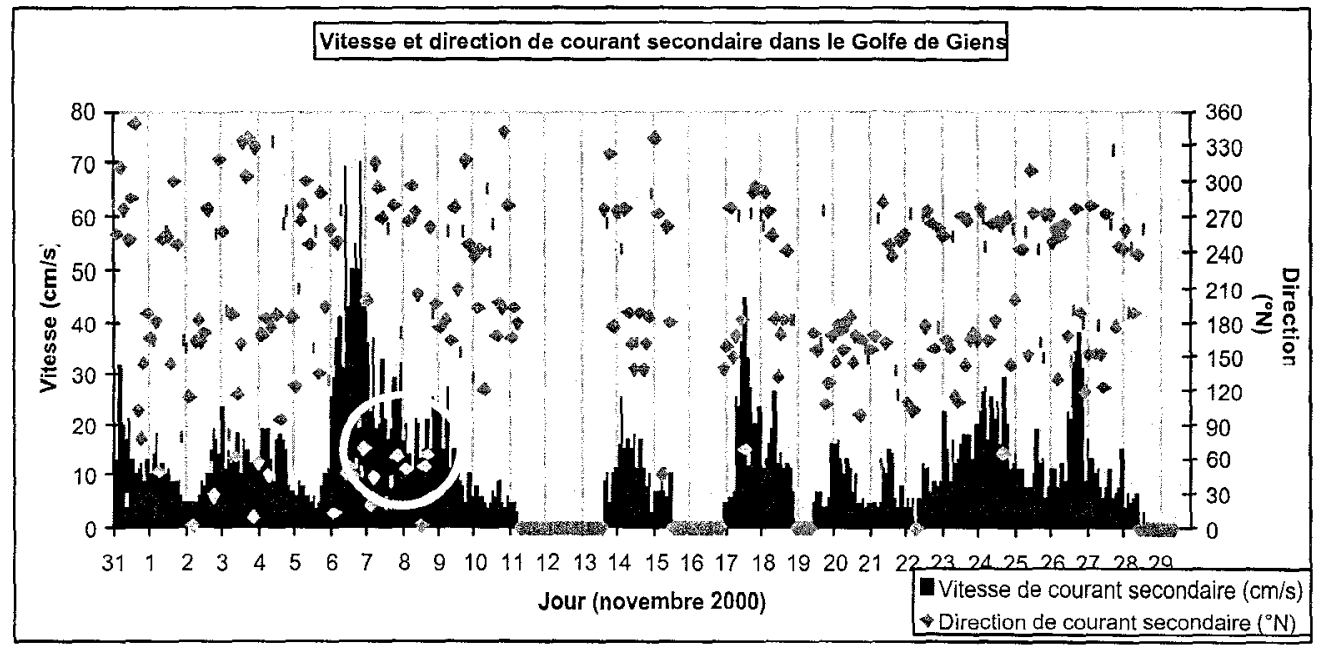

Figure 6: Vitesse et direction du courant de houle orbital secondaire au mois de novembre 2000

Figure 6 : orbital wave and current in november 2000 
Lors des périodes de forte agitation, on identifie un courant de retour vers l'Ouest dont la vitesse atteint entre $40 \mathrm{~cm} / \mathrm{s}$ et $60 \mathrm{~cm} / \mathrm{s}$. Le maximum de $70 \mathrm{~cm} / \mathrm{s}$ est atteint le 6 novembre à 10:00 et à $20: 00$, avec une orientation opposée au jet de rive $45^{\circ} \mathrm{N}$ (Figure 6). C'est la manifestation d'un fort courant de décharge déjà décrit par BLANC [1] mais jamais mesuré dans le golfe de Giens. Ces vitesses relativement élevées à $200 \mathrm{~m}$ du trait de côte sont suffisantes pour provoquer une érosion des fonds.

\subsection{Modèle d'agitation}

\subsubsection{Méthodologie}

Le modèle mis en oevre est un modèle de diffraction-réfraction elliptique (équation de Berkhoff complète résolue par une méthode d'éléments finis) qui prend en compte le transfert des informations dans tous les sens et leurs interactions. Ce modèle est adapté car il permet d'analyser l'agitation locale. à l'étude des interférences entre la côte et les ouvrages de génie côtier ou les structures marines. Il prend en compte la zone entourant le site d'étude de la plage de l'Almanarre (Figure 1). Les conditions de forçage sont définies à partir des résultats du modèle d'approche et à partir des mesures de houle. Le modèle d'éléments finis comprend 21525 noads et 23443 éléments dont la longueur caractéristique est de 7.5 mètres. Le rayon du cercle de la frontière fluide est de 3500 mètres et la surface du modèle est de 385 hectares.

\subsubsection{Résultats du modèle d'agitation}

Le première approche vise à identifier les cas d'agitations les plus contraignants en faisant varier deux paramètres : la période et la direction de la houle.

Les calculs ont été effectués sur 21 périodes allant de $5 \mathrm{~s}$ à $15 \mathrm{~s}$.

\begin{tabular}{|c|c|c|c|c|}
\hline Incidence & Ouest & $\begin{array}{c}\text { Ouest Sud- } \\
\text { Ouest } \\
247.5^{\circ}\end{array}$ & Sud-Ouest & Sud Sud-Ouest \\
\cline { 1 - 4 } Période & $270.0^{\circ}$ & $225.0^{\circ}$ & 202.5 \\
\hline $5.0 \mathrm{~s}$ & $0.0-0.5$ & $0.5-1.0$ & $0.0-0.5$ & $0.5-1.0$ \\
$5.5 \mathrm{~s}$ & $0.0-0.5$ & $0.5-1.0$ & $0.5-1.0$ & $0.5-1.0$ \\
$6.0 \mathrm{~s}$ & $0.0-0.5$ & $1.0-1.5$ & $0.5-1.0$ & $0.5-1.0$ \\
$6.5 \mathrm{~s}$ & $1.5-2.0$ & $1.5-2.0$ & $2.0-2.5$ & $2.0-2.5$ \\
$7.0 \mathrm{~s}$ & $2.0-2.5$ & $3.5-4.0$ & $2.0-2.5$ & $1.5-2.0$ \\
$7.5 \mathrm{~s}$ & $2.0-2.5$ & $1.5-2.0$ & $2.0-2.5$ & $1.0-1.5$ \\
$8.0 \mathrm{~s}$ & $0.0-0.5$ & $0.5-1.0$ & $0.5-1.0$ & $0.5-1.0$ \\
$9.0 \mathrm{~s}$ & $0.0-0.5$ & $0.5-1.0$ & $0.5-1.0$ & $0.0-0.5$ \\
$10.0 \mathrm{~s}$ & $0.0-0.5$ & $0.5-1.0$ & $0.5-1.0$ & $0.0-0.5$ \\
\hline
\end{tabular}

Tableau 1 : Coefficients d'amplifications moyens sur la zone d'étude

Table 1 : amplification coeffficient wave in the study area 
Le Tableau 1 rassemble les coefficients d'amplification moyens sur la zone d'étude. Ces résultats mettent en évidence une très sensible concentration d'énergie pour des périodes comprises entre 6.0 et 7.5 secondes, et plus particulièrement pour les incidences de secteur Ouest à Sud-Ouest. Dans une profondeur moyenne de 3 mètres d'eau, ces périodes correspondent à des longueurs d'onde de 30 à 40 mètres. On identifie sur la Figure 7 une focalisation de l'énergie qui renforce localement le déferlement sur la plage par un couloir normal à cette dernière, un peu comme dans un "guide d'onde"
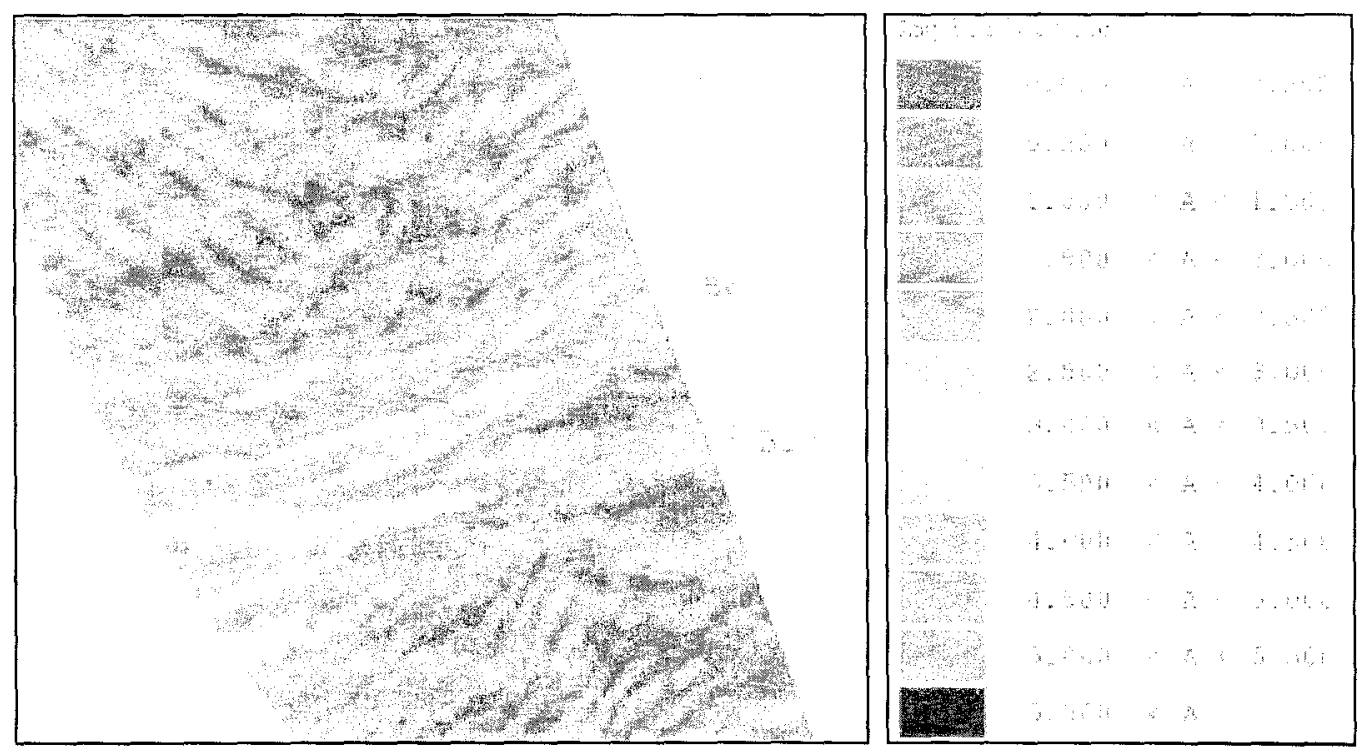

Figure 7 : Test d'agitation pour T:7s et incidence Ouest-Sud-Ouest

Figure 7 : wave simulation with $T=7 \mathrm{~s}$, è : WSW

\subsection{Capacité de transport sédimentaire de la houle}

Les résultats hydrodynamiques du modèle d'agitation permettent de déterminer la capacité mobilisatrice, en considérant que les stocks sédimentaires existent sous une forme disponible. Le Tableau 2 indique la composition granulométrique des échantillons considérés comme représentatifs de la plage de l'Almanarre pour l'évaluation du transport sédimentaire.

\begin{tabular}{|c|c|c|c|c|}
\hline $\begin{array}{c}\text { Limon } \\
<130 \mu \mathrm{m}\end{array}$ & $\begin{array}{c}\text { Sable fin } \\
130 \mu \mathrm{m}-250 \mu \mathrm{m}\end{array}$ & $\begin{array}{c}\text { Sable moyen } \\
250 \mu \mathrm{m}-500 \mu \mathrm{m}\end{array}$ & $\begin{array}{c}\text { Sable grossier } \\
500 \mu \mathrm{m}-1.6 \mathrm{~mm}\end{array}$ & $\begin{array}{c}\text { Gravier et galet } \\
1.6 \mathrm{~mm}<\end{array}$ \\
\hline $1.0 \%$ & $2.0 \%$ & $7.0 \%$ & $7.0 \%$ & $83.0 \%$ \\
\hline
\end{tabular}

Tableau 2: Répartition granulométrique des sédiments de la plage de l'Almanarre.

Table 2 : Granulometric distribution in the sediment of Almanarre Beach 
Les calculs sont effectués au moyen de la formule de Bijker-Frijlink, pondérée en fonction des probabilités associées aux différentes caractéristiques des houles (hauteur, période, incidence). Les résultats sont rapportés à une capacité de transport par unité de surface. En supposant que la dimension médiane des graviers et galets est $10 \mathrm{~mm}$, et que la plage a une largeur de 20 à 25 mètres, le transit longitudinal est de l'ordre de 16 000 à $20000 \mathrm{~m}^{3} / \mathrm{an}$ et le transit transversal dans le profil est de l'ordre de $2600 \mathrm{~m}^{3} / \mathrm{an}$ et par mètre. La résultante des transports longitudinaux est orientée vers le sud avec un débit moyen estimé à 4000m3/an dans le secteur compris entre les bornes 8 à 10 .

\subsection{Conclusion}

Cette communication a pour principal objectif de mettre en évidence les causes d'un déséquilibre hydrosédimentaire sur le secteur Nord la plage de l'Almanarre afin de pouvoir apporter des remèdes appropriés.

1. A l'origine, la brèche et les trous ne sont pas des formes reliques; elles se sont creusées avec le temps comme le démontre la comparaison des levers bathymétriques actuels et ceux du début du siècle. Cependant, les extractions de sédiments du début du siècle ont sans doute contribué à la mise à nu des couches sous-jacentes (platier gréseux et formations argileuses) et à leur démantèlement progressif, par érosion mécanique et biodégradation des grés, puis par la remise en suspension des formations argileuses sous-jacentes. Le creusement de la brèche se poursuit actuellement en profondeur et latéralement sans que l'on puisse présager d'un ralentissement ou d'une stabilisation de cette évolution.

2. La houle est le principal agent responsable des évolutions observées. Les courants induits, et en particulier le courant de retour, entrainent les sédiments de la plage vers les trous et la brèche lors des tempêtes. Les sédiments sont alors isolés du prisme littoral, car la pente est trop forte pour permettre un rééquilibrage dans le profil de la plage. L'existence d'un courant de retour est démontrée par les mesures de houle. L'autre courant qui contribue au phénomène d'érosion est le jet de rive qui transporte les sédiments de la plage dans le canal au-travers de la route du sel.

3. Les modèles d'agitation montrent que, pour certaines conditions de houle $(\grave{\mathrm{e}}=$ $250^{\circ} \mathrm{N}$ et $\mathrm{Ts}=7-8 \mathrm{~s}$ ), l'existence d'un coefficient d'amplification important entre les bornes 8 et 10 . Sans mettre directement en évidence l'existence de courants de retour, les modèles font ressortir un phénomène de «guide d'onde » qui contribue au renforcement de l'agitation dans ce secteur et ceci pour les conditions d'agitation les plus fréquentes (30j dans l'année). 
4. l'ordre de grandeur de la capacité de transport est de $4000 \mathrm{~m} 3 / \mathrm{an}$ dans le secteur de la brèche pour un transit potentiel estimé entre 15 et $20000 \mathrm{~m} 3 \mathrm{sur}$ l'ensemble du secteur Nord du tombolo Ouest de Giens.

$\mathrm{Au}$ vu de ces résultats, les interventions envisagées consisteraient à reconstituer le profil de plage par un rechargement de sédiments et à renforcer le platier gréseux instable par une butée de pied dont la nature est envisagée sous la forme de tubes géotextiles remplis de sable. Cette solution est privilégiée en raison de son caractère réversible.

\section{Remerciements}

Nous tenons à remercier M. GONZALES, Mme GADAUD, M. BRUNO de la Ville d'Hyères, M. LIEUTAUD du service maritime (DDE83), M. SERANTONI (Eol), GOASGUEN (CETMEF) pour leur aide et la documentation fournie.

\section{Bibliographie}

[1] J.J.BLANC - 1975 Recherches de sédimentologie appliquée au littoral rocheux de la Provence-Aménagement et protection -CNEXO 1975

[2]CETMEF - 1999 Données de houle: mesures au large de Porquerolles. Période 14/05/92 -16/06/95 - Période de 1992-1999 enregistrement de coups de mer

[3]ERG 2001 Levé géologique et géotechnique du tombolo Ouest de Gienssecteur Nord (Hyères Var) 